\title{
Rare $Z$ boson decays to a hidden sector
}

\author{
Nikita Blinov, ${ }^{1}$ Eder Izaguirre, ${ }^{2}$ and Brian Shuve ${ }^{3,1}$ \\ ${ }^{1}$ SLAC National Accelerator Laboratory, 2575 Sand Hill Rd., Menlo Park, California 94025, USA \\ ${ }^{2}$ Brookhaven National Laboratory, Upton, New York 11973, USA \\ ${ }^{3}$ Harvey Mudd College, 301 Platt Blvd., Claremont, California 91711, USA
}

(Received 11 November 2017; published 18 January 2018)

\begin{abstract}
We demonstrate that rare decays of the Standard Model $Z$ boson can be used to discover and characterize the nature of new hidden-sector particles. We propose new searches for these particles in soft, highmultiplicity leptonic final states at the Large Hadron Collider. The proposed searches are sensitive to lowmass particles produced in $Z$ decays, and we argue that these striking signatures can shed light on the hidden-sector couplings and mechanism for mass generation.
\end{abstract}

DOI: 10.1103/PhysRevD.97.015009

\section{INTRODUCTION}

The Large Hadron Collider (LHC) is now probing the Standard Model (SM) and its possible extensions at energies exceeding the electroweak scale. The lack of any definitive discoveries beyond the Higgs boson, however, suggests that new physics within the reach of the LHC may appear in unexpected places. It is now widely appreciated that some classes of signatures are challenging to probe at a hadron collider. These include new electroweak multiplets with masses near the electroweak scale [1-4]; particles produced via the strong interactions that decay to approximately degenerate states [5]; "stealth" spectra [6]; or all-hadronic final states [7].

In this paper, we focus on searches for low-mass particles, which can likewise be difficult to find at the LHC. The signatures of new light particles are manifold and are sensitive to both the manner of production (i.e., whether the low-mass states are produced directly or in the decays of heavier particles), as well as whether there exists a single new particle [8,9] or an entire hidden sector or "hidden valley" [10-12]. When the new low-mass particles decay back into SM final states, the result is typically a high-multiplicity final state $[10,12-14]$. Since the energy in the event is divided among many particles, the final-state particles are typically soft and could be lost in the enormous multijet backgrounds or contaminated by pileup. However, hidden sectors can also give rise to spectacular signatures such as high multiplicities of leptons, displaced vertices, and other nonstandard phenomena [10-12,15-17]. These

Published by the American Physical Society under the terms of the Creative Commons Attribution 4.0 International license. Further distribution of this work must maintain attribution to the author(s) and the published article's title, journal citation, and DOI. Funded by SCOAP ${ }^{3}$. can be used to suppress the sizable SM backgrounds, provided the signals are sufficiently energetic to be efficiently reconstructed.

Because of its high luminosity, the LHC is an exquisite tool for studying hidden-sector particles that are too heavy to be produced in other intensity-frontier experiments. Through Phase II, the LHC will produce unprecedented numbers of electroweak and Higgs bosons, exceeding the statistics from LEP by many orders of magnitude. By looking for new states produced in the rare decays of SM particles, we may probe ever-smaller couplings between SM particles and hidden sectors. The production of hiddensector particles in the decays of the SM Higgs boson has been well studied (see, for example, Ref. [18]). In this study, we focus on rare $Z$ boson decays, which are most important in vector-portal models of hidden sectors.

To achieve optimal sensitivity to hidden sectors at the LHC, it is essential that the experiments are able to efficiently trigger on and reconstruct new low-mass objects. Dedicated search and reconstruction strategies are needed to uncover evidence for low-mass particles, especially if the particles are boosted and fail conventional isolation criteria. Such strategies have been successfully implemented in several contexts, such as for lepton jets [19-21]; compressed stops [22,23]; long-lived, low-mass particles decaying in the ATLAS hadronic calorimeter and muon spectrometer [24]; and searches by the LHCb Collaboration for long-lived particles in the forward direction [25]. However, comprehensive studies are needed to ensure that signals are not lost at trigger or event reconstruction level, and we undertake such a study with a focus on highmultiplicity (6+ particle) decays of the $Z$ boson into hidden sector particles.

We take as a concrete example a minimal hidden sector with a spontaneously broken Abelian gauge interaction $[26,27]$. The new gauge boson (the dark photon, $A^{\prime}$ ) 
couples to the SM via kinetic mixing with the hypercharge boson, resulting in $Z$ decays into the hidden sector. We show that $Z$ decays into $A^{\prime}$ in association with the dark Higgs boson, $h_{\mathrm{D}}$, lead to striking signatures such as multiple resonances, high multiplicities of soft leptons, and long-lived particles. The decay $Z \rightarrow A^{\prime} h_{\mathrm{D}}$ is a dark version of the Higgs-strahlung process and additionally allows for a direct test of the mass generation mechanism in the hidden sector. If the dark Higgs decays via $h_{\mathrm{D}} \rightarrow A^{\prime} A^{\prime}$ as illustrated in Fig. 1, then $A^{\prime}$ decays into SM particles can yield a final state with as many as six leptons.

We show that searches for dark Higgs-strahlung in rare $Z$ decays can be competitive with and exceed the sensitivity of existing and proposed search strategies for dark gauge and Higgs bosons, while also yielding new information. Because the decay $Z \rightarrow A^{\prime} h_{\mathrm{D}}$ is sensitive to the hiddensector gauge coupling, $\alpha_{\mathrm{D}}$, a discovery of a signal in this process provides complementary information to direct searches for $A^{\prime}$. Previous proposals entailed looking for $A^{\prime}$ at the LHC via its contribution to Drell-Yan production above $10 \mathrm{GeV}[28,29]$, in rare 3-body $Z$ decays into leptons and a dark gauge boson [30], as well as in decays of the SM Higgs boson [18,31-33]. Finally, Ref. [34] evaluated the prospects for discovering $A^{\prime}$ and $h_{\mathrm{D}}$ via Higgs-strahlung at future lepton colliders, where they focused on decays to invisible hidden-sector states.

While our study is phenomenologically driven, we demonstrate sensitivity to models of hidden sectors that are well motivated by various shortcomings of the SM, especially the need to account for dark matter but also potentially outstanding problems in neutrino physics and other areas. Indeed, low-mass dark matter scenarios are typically only viable with additional low-mass mediators in the hidden sector $[8,35,36]$. Hidden sectors can also generically arise in ultraviolet completions of the SM such as string theory [37-39].

We outline our benchmark hidden-sector model in Sec. II. We then enumerate the signatures of rare $Z$ decay into the hidden sector, focusing on signals with high lepton

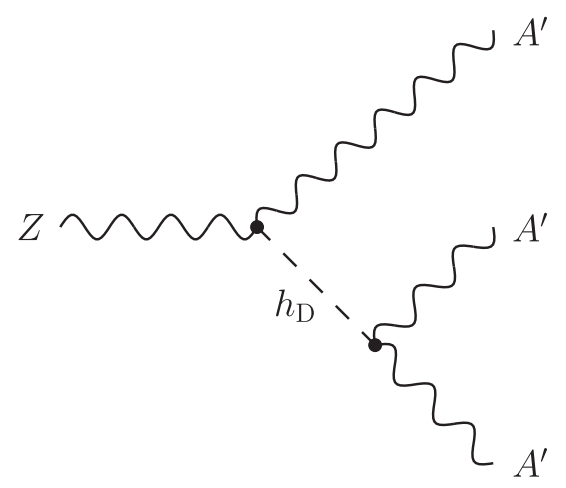

FIG. 1. Feynman diagram illustrating dark Higgs $\left(h_{\mathrm{D}}\right)$ and dark photon $\left(A^{\prime}\right)$ production in rare $Z$ boson decays. The dark photons in $h_{\mathrm{D}}$ decay can be on or off shell. multiplicities and hidden-sector resonances. We give projected LHC sensitivities to prompt hidden-sector signals in Sec. III, and we discuss displaced signals in Sec. IV. Our outlook is given in Sec. V.

\section{AN ABELIAN HIDDEN SECTOR}

The benchmark model we consider is one of the simplest examples of a hidden sector: a minimal $U(1)_{\mathrm{D}}$ gauge interaction spontaneously broken by a nondecoupled Higgs field. The model is specified by the following Lagrangian:

$$
\begin{aligned}
\mathcal{L} \supset & -\frac{1}{4} F_{\mu \nu}^{\prime} F^{\prime \mu \nu}+\frac{\varepsilon_{Y}}{2} F_{\mu \nu}^{\prime} B^{\mu \nu} \\
& +\left|\partial_{\mu} H_{\mathrm{D}}-i e_{\mathrm{D}} A_{\mu}^{\prime} H_{\mathrm{D}}\right|^{2}-V\left(H, H_{\mathrm{D}}\right),
\end{aligned}
$$

where $F^{\prime \mu \nu}\left(B^{\mu \nu}\right)$ is the $U(1)_{\mathrm{D}}$ (hypercharge) field strength, $e_{\mathrm{D}}=\sqrt{4 \pi \alpha_{\mathrm{D}}}$ is the $U(1)_{\mathrm{D}}$ gauge coupling, and $V$ is the scalar potential

$$
\begin{aligned}
V\left(H, H_{\mathrm{D}}\right)= & -\mu_{H}^{2}|H|^{2}-\mu_{H_{\mathrm{D}}}^{2}\left|H_{\mathrm{D}}\right|^{2}+\kappa|H|^{2}\left|H_{\mathrm{D}}\right|^{2} \\
& +\lambda|H|^{4}+\lambda_{\mathrm{D}}\left|H_{\mathrm{D}}\right|^{4} .
\end{aligned}
$$

The SM and dark Higgs acquire vacuum expectation values (VEVs) $\langle H\rangle=(0, v / \sqrt{2})$ and $\left\langle H_{\mathrm{D}}\right\rangle=v_{\mathrm{D}} / \sqrt{2}$, breaking the electroweak and $U(1)_{\mathrm{D}}$ gauge symmetries, respectively. As a result, gauge eigenstates undergo mixing [26]. For a recent review of the model, see Ref. [32].

The kinetic and mass terms for the gauge bosons can be simultaneously diagonalized using the transformation (to leading order in $\varepsilon_{Y}$ and $\theta_{Z}$ )

$$
\begin{aligned}
Z_{\mu} \rightarrow Z_{\mu} & -\left(\theta_{Z}+\varepsilon \tan \theta_{\mathrm{W}}\right) A_{\mu}^{\prime}, \\
A_{\mu}^{\prime} & \rightarrow A_{\mu}^{\prime}+\theta_{Z} Z_{\mu}, \\
A_{\mu} & \rightarrow A_{\mu}+\varepsilon A_{\mu}^{\prime},
\end{aligned}
$$

where $\varepsilon \equiv \varepsilon_{Y} \cos \theta_{\mathrm{W}}, \theta_{\mathrm{W}}$ is the weak mixing angle, and

$$
\theta_{Z}=-\frac{\varepsilon \tan \theta_{\mathrm{W}} m_{Z}^{2}}{m_{Z}^{2}-m_{A^{\prime}}^{2}}+\mathcal{O}\left(\varepsilon^{3}\right)
$$

is the gauge-boson mixing angle. The $Z$ and $A^{\prime}$ bosons have masses equal to the unshifted values at $\mathcal{O}(\varepsilon)$, while the photon $A$ remains exactly massless.

The scalar states can also undergo mixing after gaugesymmetry breaking. The mass eigenstates are

$$
\left(\begin{array}{c}
h \\
h_{\mathrm{D}}
\end{array}\right)=\left(\begin{array}{cc}
\cos \theta_{h} & -\sin \theta_{h} \\
\sin \theta_{h} & \cos \theta_{h}
\end{array}\right)\left(\begin{array}{c}
h^{(0)} \\
h_{D}^{(0)}
\end{array}\right)
$$

where $h^{(0)}, h_{\mathrm{D}}^{(0)}$ are the $C P$-even gauge eigenstate components of $H$ and $H_{\mathrm{D}}$, respectively. In the limit of small mixed quartic coupling $\kappa$, the $h-h_{\mathrm{D}}$ mixing angle is given by 


$$
\sin \theta_{h} \approx \frac{\kappa}{2} \frac{v v_{\mathrm{D}}}{\lambda_{\mathrm{D}} v_{\mathrm{D}}^{2}-\lambda v^{2}}
$$

Except where otherwise noted, we assume that the dominant hidden-sector portal to the SM is via the coupling between gauge bosons, $\varepsilon$.

Because the $U(1)_{\mathrm{D}}$ gauge symmetry is spontaneously broken, the masses of hidden-sector particles are related to the symmetry-breaking parameter. In particular, the dark Higgs VEV gives a mass to the dark gauge boson, leading to a dark Higgs-strahlung $h_{\mathrm{D}}-A^{\prime}-A^{\prime}$ vertex by analogy with the symmetry breaking pattern in the SM. After the vector and scalar eigenstates mix, we obtain the following mass-basis Lagrangian:

$\mathcal{L} \supset g_{h_{\mathrm{D}} A^{\prime} Z} h_{\mathrm{D}} A_{\mu}^{\prime} Z^{\mu}+g_{h_{\mathrm{D}} A^{\prime} A^{\prime}} h_{\mathrm{D}} A_{\mu}^{\prime} A^{\prime \mu}+g_{A^{\prime} \bar{f} f} A_{\mu}^{\prime} \bar{f} \gamma^{\mu} f$,

along with additional terms that are not relevant for the phenomenology we study. The approximate couplings may be expressed simply in the $m_{A^{\prime}} \ll m_{Z}$ limit,

$$
\begin{gathered}
g_{h_{\mathrm{D}} A^{\prime} Z}=2 e_{\mathrm{D}} \theta_{Z} \cos \theta_{h} m_{A^{\prime}}, \\
g_{h_{\mathrm{D}} A^{\prime} A^{\prime}}=e_{\mathrm{D}}^{2} v_{\mathrm{D}}, \\
g_{A^{\prime} f f}=\varepsilon e Q_{f} .
\end{gathered}
$$

The first term in Eq. (9) gives rise to $Z$ boson decay into $h_{\mathrm{D}}$ and $A^{\prime}$ with rate

$$
\begin{aligned}
\Gamma_{Z \rightarrow A^{\prime} h_{\mathrm{D}}}= & \frac{2 \alpha_{\mathrm{D}} \theta_{Z}^{2} \cos ^{2} \theta_{h} m_{A^{\prime}}^{2}}{3 m_{Z}} \\
& \times\left[1+\frac{\left(m_{Z}^{2}+m_{A^{\prime}}^{2}-m_{h_{\mathrm{D}}}^{2}\right)^{2}}{8 m_{Z}^{2} m_{A^{\prime}}^{2}}\right] \beta\left(\frac{m_{A^{\prime}}}{m_{Z}}, \frac{m_{h_{\mathrm{D}}}}{m_{Z}}\right),
\end{aligned}
$$

where $\beta(x, y)=\left[\left(1-(x-y)^{2}\right)\left(1-(x+y)^{2}\right)\right]^{1 / 2}$. This rate vanishes as $m_{A^{\prime}} \rightarrow 0$, but becomes appreciable as $m_{A^{\prime}}$ increases above $\sim 10 \mathrm{GeV}$, which is precisely where constraints from low-energy colliders become ineffective.

The decays of the $A^{\prime}$ and the $h_{\mathrm{D}}$ depend on the spectrum of the hidden sector. When $A^{\prime}$ and $h_{\mathrm{D}}$ are the lightest states, they decay back into SM particles. The $A^{\prime}$ decays dominantly into electrically charged SM fermion pairs via the coupling in Eq. (12). When kinematically allowed, the dark Higgs can decay into one or two on-shell $A^{\prime}$ via $h_{\mathrm{D}} \rightarrow A^{\prime} A^{\prime(*)}$, and the $A^{\prime}$ in turn decays into SM fermions. Finally, if $m_{h_{\mathrm{D}}}<m_{A^{\prime}}$, then the $h_{\mathrm{D}}$ can decay via two offshell $A^{\prime}$, although radiative corrections typically induce a comparable decay via scalar mixing.

Having defined the model, we can consider new search strategies for discovering the hidden sector through the process, $Z \rightarrow A^{\prime} h_{\mathrm{D}}$. The specific signatures depend on the mass hierarchy of the $A^{\prime}$ and $h_{\mathrm{D}}$. For $m_{A^{\prime}}<m_{h_{\mathrm{D}}}$, the decay
$h_{\mathrm{D}} \rightarrow A^{\prime} A^{\prime(*)}$ is kinematically allowed and occurs relatively rapidly, giving rise to prompt signals. We propose searches for such prompt signatures in Sec. III. If instead $m_{A^{\prime}}>m_{h_{\mathrm{D}}}$, then the $h_{\mathrm{D}}$ lifetime is typically long; we explore this scenario in Sec. IV. Before we study the $Z \rightarrow A^{\prime} h_{\mathrm{D}}$ signal, however, we first summarize existing constraints on the model.

\section{A. Existing constraints}

We focus on masses $m_{A^{\prime}}, m_{h_{\mathrm{D}}} \gtrsim 1 \mathrm{GeV}$ which are most relevant for the LHC.

Low-energy $e^{+} e^{-}$colliders: The clean environment and high luminosities of low-energy $e^{+} e^{-}$colliders allow for powerful searches for $A^{\prime}$ and $h_{\mathrm{D}}$ for masses below $10 \mathrm{GeV}$. The kinetic-mixing coupling of $A^{\prime}$ to fermions is studied in the radiative return reaction $e^{+} e^{-} \rightarrow A^{\prime} \gamma, A^{\prime} \rightarrow \ell^{+} \ell^{-}$for electron and muon final states. In particular, the analysis from Ref. [40] puts mass-dependent upper bounds on $\varepsilon$ in the range $3 \times 10^{-4}-10^{-3}$ for masses $m_{A^{\prime}} \lesssim 10 \mathrm{GeV}$. $B A B A R$ and Belle also constrain hidden sectors through searches for the dark Higgs-strahlung process, $e^{+} e^{-} \rightarrow$ $A^{\prime} h_{\mathrm{D}}[41,42]$. These searches constrain the combination of couplings $\alpha_{\mathrm{D}} \varepsilon^{2} \lesssim 10^{-9}$, with the precise limits depending on $m_{A^{\prime}}$ and $m_{h_{\mathrm{D}}}$. Belle II is expected to significantly extend this sensitivity to hidden sectors below $10 \mathrm{GeV}$, although in all cases $B$ factories lose sensitivity for $m_{A^{\prime}}>10 \mathrm{GeV}$.

Electroweak precision observables: Precision tests of electroweak symmetry breaking set stringent bounds for $m_{A^{\prime}}<m_{Z}$. Bounds from electroweak precision observables (EWPO) arise predominantly from the mass mixing of $A^{\prime}$ with $Z$, although other EWPO observables get modified by virtue of the fact that electrically charged SM particles acquire an effective millicharge under the $A^{\prime}$ (see, for instance, Ref. [43] and a newer analysis including prospects for future improvements in EWPO at high-energy lepton colliders by Ref. [32]). Away from $m_{A^{\prime}}=m_{Z}$, the EWPO constraint is driven primarily by the tree-level $\varepsilon$-dependent shift to $m_{Z}$ relative to the SM value, $m_{Z, 0}$, which enters the prediction of $m_{W}[32,43]$. The resulting bound can be estimated as $[32,44]$

$$
\frac{m_{Z}-m_{Z, 0}}{m_{Z, 0}} \approx \frac{\varepsilon^{2} \tan ^{2} \theta_{\mathrm{W}} m_{Z}^{2}}{2\left(m_{Z}^{2}-m_{A^{\prime}}^{2}\right)}<9 \times 10^{-5} .
$$

For $m_{A^{\prime}} \ll m_{Z}$, the mass shift is independent of $m_{A^{\prime}}$ and constrains $\varepsilon \gtrsim 3 \times 10^{-2}$.

LHC constraints: The $A^{\prime}$ mediates new contributions to Drell-Yan production of leptons at the LHC. A reinterpretation of a CMS study of the Drell-Yan process at $\sqrt{s}=$ $7 \mathrm{TeV}$ [45] sets constraints on the $A^{\prime}$ mass via a dilepton resonance search [28] (see Ref. [28] and an updated analysis by Ref. [32] on future prospects for Drell-Yan sensitivity to $A^{\prime}$ ). Dark photons heavier than the $Z$ have 
been constrained by recasting ATLAS and CMS searches for heavy $Z^{\prime}$ [46]. A recent $\mathrm{LHCb}$ search for inclusive Drell-Yan production of dark photons has set powerful constraints in the $m_{A^{\prime}} \sim 10-70 \mathrm{GeV}$ mass range with $1.5 \mathrm{fb}^{-1}$ of data at $\sqrt{s}=13 \mathrm{TeV}$ [47]. In the case where $h_{\mathrm{D}}$ has an appreciable mixing with $h_{\mathrm{SM}}$, there are also constraints on the hidden sector via $h_{\mathrm{SM}} \rightarrow A^{\prime} A^{\prime}[32,48]$; in the limit of small scalar mixing that is our focus, the latter searches do not apply any meaningful constraint.

\section{PROMPT MULTILEPTON SIGNALS FROM RARE $Z$ DECAYS}

We have argued that the dark sector benchmark model in Eq. (1) gives rise to striking signatures in the decay of the SM $Z$ boson via $Z \rightarrow A^{\prime} h_{\mathrm{D}}$. Studying this process would allow for not only the discovery of $A^{\prime}$ and $h_{\mathrm{D}}$ but also can provide information about the hidden sector couplings and mass generation mechanisms. We now examine in detail the phenomenology of this rare $Z$ decay.

Fully on-shell decays: If $m_{h_{\mathrm{D}}}>2 m_{A^{\prime}}$, then the following decay chain occurs:

$$
p p \rightarrow Z \rightarrow A^{\prime} h_{\mathrm{D}} \rightarrow A^{\prime} A^{\prime} A^{\prime}
$$

The $A^{\prime}$ can decay to various SM final particles. In principle, this gives final-state signatures with three $A^{\prime}$ resonances, as well as the intermediate $h_{\mathrm{D}}$ resonance. In the case of fully leptonic decays, this is an extremely clean and spectacular signature. In practice, other $Z$ decays may give better model sensitivity because of the small probability that each $A^{\prime}$ decays leptonically, as well as the efficiency penalties associated with reconstructing all six soft leptons. Combinatorics may also present a challenge to reconstructing the signal. However, even the more inclusive case with $Z \rightarrow h_{\mathrm{D}} A^{\prime}, h_{\mathrm{D}} \rightarrow 4 \ell$ (with the other $A^{\prime}$ decaying to arbitrary final states) features two leptonically decaying $A^{\prime}$ resonances that together reconstruct the $h_{\mathrm{D}}$. We therefore investigate signatures with at least four leptons from the $h_{\mathrm{D}}$ decay.

Because of the limited available phase space in six-body $Z$ decays, it is beneficial to use multilepton triggers with low $p_{\mathrm{T}}$ thresholds. The current triggers that best fit these requirements are the three-lepton triggers. For ATLAS, these are [49]

(i) three loose $e$ 's: $p_{\mathrm{T}} \geq 15,8,8 \mathrm{GeV}$ at $\mathrm{L} 1(17,10,10$ at high-level trigger (HLT)),

(ii) three $\mu$ 's: $p_{\mathrm{T}}>6 \mathrm{GeV}(3 \times 6$ at HLT $)$.

For CMS, we examine the thresholds from a multilepton analysis such as $Z Z \rightarrow 4 \ell$ [50], which used

(i) three $e$ 's: $p_{\mathrm{T}} \geq 15,8,5 \mathrm{GeV}$.

While this analysis was conducted at $\sqrt{s}=8 \mathrm{TeV}$, the CMS dilepton trigger thresholds did not increase appreciably between $8 \mathrm{TeV}$ and $13 \mathrm{TeV}$ [51,52], and we use this as a proxy for what can currently be expected in terms of trigger thresholds. We note that trilepton thresholds may go up in the high-luminosity phase of the LHC; however, in this case 4-lepton triggers could alternatively be used to keep $p_{\mathrm{T}}$ thresholds low.

Partially off-shell decays: When $m_{A^{\prime}}<m_{h_{\mathrm{D}}}<2 m_{A^{\prime}}$, the decay $h_{\mathrm{D}} \rightarrow A^{\prime} A^{\prime}$ is kinematically forbidden, but the semi-off-shell decay $h_{\mathrm{D}} \rightarrow A^{\prime} A^{\prime *} \rightarrow A^{\prime} \bar{f} f$ (where $f$ is a SM fermion) can occur. While $\Gamma_{h_{\mathrm{D}}}$ is suppressed by a factor of $\varepsilon^{2}$ for semi-off-shell decays compared to the case of fully on-shell decays, we find that the decay still occurs promptly for the parameters of relevance to the LHC. Thus, the reaction proceeds as follows:

$$
p p \rightarrow Z \rightarrow A^{\prime} h_{\mathrm{D}} \rightarrow A^{\prime} A^{\prime} \bar{f} f .
$$

The principal difference between the semi-off-shell and fully on-shell decays is that one of the pairs of SM particles no longer reconstructs a resonance. As we shall soon see, however, the backgrounds are sufficiently low that we do not necessarily need to impose a $m_{A^{\prime}}$ resonance reconstruction requirement on the leptons within $h_{\mathrm{D}} \rightarrow 4 \ell$ decays, and so the fully and partially on-shell $h_{\mathrm{D}}$ decays can be studied simultaneously. Depending on how actual experimental conditions compare to Monte Carlo simulations, these two cases may need to be studied separately.

Simulations: In performing our analysis, we generate parton-level events at leading order with MadGraph5 aMC@NLO [53]. To capture the effects of initial-state radiation on signal acceptance, we generate events with up to one extra parton and match them to the showered events using the shower- $k_{\mathrm{T}}$ scheme [54]. We use the parton shower program Pythia 8.2 [55-57].

Reinterpretation of existing searches: Existing searches by ATLAS and CMS are already sensitive to signals with high lepton multiplicities. For example, CMS has a search for new electroweak supersymmetric (SUSY) particles that decay to three or more leptons [52]. We reinterpret the results of the low- $\mathbb{E}_{\mathrm{T}}$, 4-lepton signal regions in terms of our signal model. In order to derive the constraints from the CMS search, we must apply lepton identification efficiencies, which are somewhat small for leptons with low $p_{\mathrm{T}}$. Because Ref. [52] only provides the low- $p_{\mathrm{T}}$ lepton tagging efficiencies for the most pessimistic working point, we must use the pessimistic values and obtain a conservative result. The true signal efficiency is almost certainly better than what we find, because Ref. [52] states that a looser set of lepton identification criteria is used for searches with four leptons, but does not specifically state what these efficiencies are.

We find that the signal region (SR) H of Ref. [52], which requires four leptons and fewer than two opposite-sign, same-flavor (OSSF) lepton pairs, is most sensitive to the hidden sector topology we study. In this case, the $Z \rightarrow$ $A^{\prime} h_{\mathrm{D}} \rightarrow 6 \ell$ decay can pass the CMS signal selections if two of the leptons are lost and only one OSSF pair is 
reconstructed. ${ }^{1}$ Using the $\mathrm{CL}_{\mathrm{s}}$ method [58], we estimate a constraint on the dark photon kinetic mixing, $\varepsilon$, at the 95\% confidence level (c.1.).

The limits from the CMS multilepton search are better than the constraints from EWPO, but somewhat worse than the recent bounds from LHCb [47], and so we do not show them explicitly. However, as argued above our reinterpretation is almost certainly more conservative than the actual analysis, and the existing CMS search may in fact place limits on the dark Abelian Higgs model that are competitive with LHCb. Crucially, the CMS search is not optimized for the hidden-sector signal, and so the fact that it is already somewhat competitive with existing dedicated searches for $A^{\prime}$ affirms the important role of rare $Z$ decays in probing hidden sectors.

Proposal for new multilepton search: Our signal features distinctive kinematics and multiple resonances in $h_{\mathrm{D}} \rightarrow$ $A^{\prime} A^{\prime} \rightarrow 4 \ell$ decays. These features can be exploited to significantly reduce both the background and the uncertainty on its estimate compared to the CMS SUSY signal regions while maximizing signal efficiency.

We define a signal region for the decay mode $Z \rightarrow A^{\prime} h_{\mathrm{D}}$, $h_{\mathrm{D}} \rightarrow A^{\prime} A^{\prime} \rightarrow 4 \ell$, while remaining agnostic about the third $A^{\prime}$ decay mode. The selections for our signal region are as follows:

(i) Trigger selection: Require four muons with $p_{\mathrm{T}}>$ $7 \mathrm{GeV}$ or any four leptons with $p_{\mathrm{T}}>15,8,7$, $5 \mathrm{GeV}$. All leptons must be in the central part of the detector $(|\eta|<2.5)$.

(ii) Isolation: Require that each lepton be isolated from hadronic activity or photons. We do this by finding the scalar sum $p_{\mathrm{T}}$ of all hadrons or photons within $\Delta R=0.3$ of the lepton and requiring that the sum be either less than $5 \mathrm{GeV}$ or less than $20 \%$ of the lepton $p_{\mathrm{T}}$.

(iii) $A^{\prime}$ signal selection: Require at least two OSSF lepton pairs (each pair forms an $A^{\prime}$ candidate).

(iv) Suppression of $Z$ backgrounds: Veto events with any OSSF dilepton pair satisfying $\left|m_{2 \ell}-m_{Z}\right|<5 \mathrm{GeV}$. Also, since the signal arises from $Z \rightarrow 4 \ell+X$ decays, veto events with any $m_{4 \ell}+5 \mathrm{GeV}>m_{Z}$.

(v) Suppression of combinatorics: When reconstructing $A^{\prime}$ candidates among possible OSSF dilepton pairs, $A^{\prime}$ candidates are chosen such that the dilepton pairs minimize $\left|m_{i}-m_{j}\right|$, where $i$ and $j$ refer to the $A^{\prime}$ candidates and $m_{i}$ and $m_{j}$ refer to their respective masses.

We find the efficiency of these selections is $\approx 20 \%-50 \%$ for signal events (depending on the masses $m_{A^{\prime}}$ and $m_{h_{\mathrm{D}}}$ ) and about $\approx 1 \%$ for background.

\footnotetext{
${ }^{1}$ There is also a signal region with two OSSF pairs (SR G) [52], which features a larger signal acceptance but significantly larger backgrounds (and systematic uncertainties). As a result, the signal sensitivity is better for SR H.
}

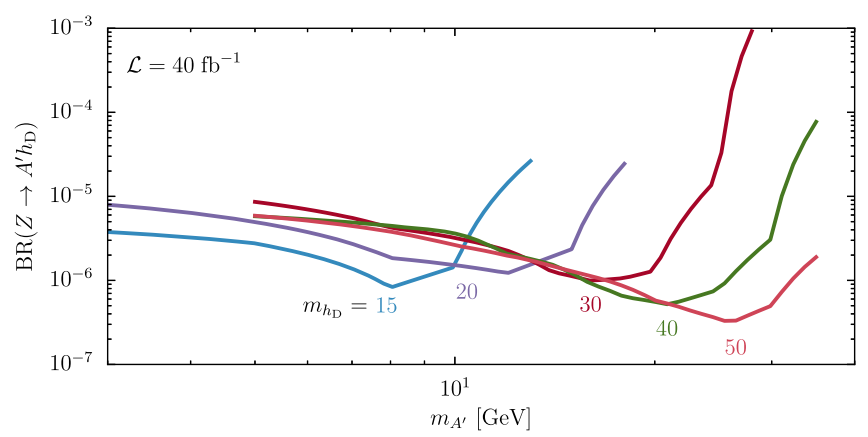

FIG. 2. Projected $95 \%$ c.l. sensitivity of an LHC search with $\mathcal{L}=40 \mathrm{fb}^{-1}$ at $\sqrt{s}=13 \mathrm{TeV}$ for $Z \rightarrow h_{\mathrm{D}} A^{\prime} \rightarrow 4 \ell+X$ where $4 \ell$ are required to reconstruct $m_{h_{\mathrm{D}}}$. The sensitivity is expressed in terms of the accessible branching fraction of $Z \rightarrow h_{\mathrm{D}} A^{\prime}$ decays.

Additionally, we exploit the fact that the four leptons in $h_{\mathrm{D}} \rightarrow 4 \ell$ decays reconstruct $m_{h_{\mathrm{D}}}$. A typical 4-lepton invariant mass resolution is approximately [59]

$$
\Delta m_{4 \ell}=0.13 \mathrm{GeV}+0.065 m_{h_{\mathrm{D}}} .
$$

Therefore, for each signal hypothesis mass $m_{h_{\mathrm{D}}}$, we define a bin centered at mass $m_{h_{\mathrm{D}}}$ and width given by Eq. (17), and consider only events inside this bin. This eliminates much of the remaining background. We comment that all of our selection criteria are approximate and should be reoptimized by the experimental collaborations once more accurate, data-driven background estimates are obtained.

Backgrounds: The dominant SM background by far is the $\gamma / Z$-initiated inclusive $p p \rightarrow 4 \ell+X$ final state. ${ }^{2}$ Other backgrounds, such as $t \bar{t}$ and multiboson production, are subdominant after the cuts we apply and could be further suppressed by additional requirements on $b$-jets, the maximum allowed lepton $p_{\mathrm{T}}$, and the maximum $\ddot{E}_{\mathrm{T}}$.

Sensitivity projections: We now make projections for the sensitivity of the above proposed search to the dark Abelian Higgs model. In addition to the selections described above, we apply a flat event-level $50 \%$ penalty for reconstructing the four soft leptons. We evaluate the maximum number of allowed signal events at $95 \%$ c.l. assuming Poisson statistics and a background-only hypothesis, and extract an estimated limit on the signal rate. We first express the expected sensitivity in terms of the $Z \rightarrow A^{\prime} h_{\mathrm{D}}$ branching fraction, which we show for $40 \mathrm{fb}^{-1}$ in Fig. 2. This sensitivity depends only on the $A^{\prime}$ and $h_{\mathrm{D}}$ masses and not separately on the hidden-sector couplings. In Fig. 3, we show the estimated sensitivity to the kinetic mixing parameter, $\varepsilon$, for various values of $\alpha_{\mathrm{D}}$. Looking forward, in Fig. 4 we demonstrate for the particular case $\alpha_{\mathrm{D}}=0.1$ the sensitivity that can be achieved if the same trigger and

\footnotetext{
${ }^{2}$ This was also found to be the dominant background in both CMS low- $E_{\mathrm{T}}$ 4-lepton searches (with either $<2$ or $=2$ OSSF pairs) [52].
} 


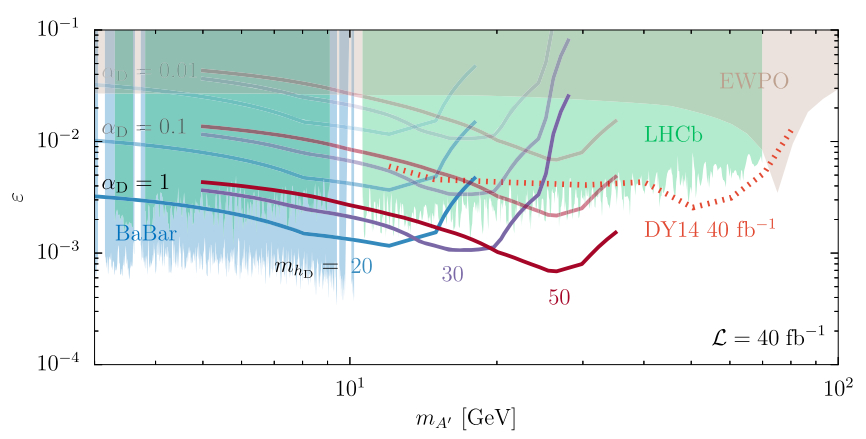

FIG. 3. Projected $95 \%$ c.l. sensitivity of an LHC search with $\mathcal{L}=40 \mathrm{fb}^{-1}$ at $\sqrt{s}=13 \mathrm{TeV}$ for $Z \rightarrow h_{\mathrm{D}} A^{\prime} \rightarrow 4 \ell+X$ where $4 \ell$ are required to reconstruct $m_{h_{\mathrm{D}}}$. Each curve is labeled by the value of $m_{h_{\mathrm{D}}}$ in $\mathrm{GeV}$. The dotted line gives the sensitivity of the Drell-Yan search from Ref. [32]. We also show existing constraints from electroweak precision observables [32], BABAR [40], and $\mathrm{LHCb}$ [47]. The three sets of lines from bottom (dark) to top (light) correspond to $\alpha_{\mathrm{D}}=1,0.1$, and 0.01 .

analysis selections can be maintained throughout Run 3 and the high-luminosity LHC integrated luminosity benchmarks. For comparison with our results, we display the projected sensitivity from dilepton resonance searches.

We emphasize that beyond allowing for a discovery of $A^{\prime}$ and $h_{\mathrm{D}}$, such a search can yield additional information about the dark sector. In particular, the $Z$ branching ratio into $A^{\prime} h_{\mathrm{D}}$ is sensitive to the dark coupling, $\alpha_{\mathrm{D}}$, which is readily seen in Fig. 3. Thus, a combined discovery of $A^{\prime}$ in Drell-Yan production direct production and via $Z \rightarrow A^{\prime} h_{\mathrm{D}}$ would allow for the determination of the hidden-sector coupling, $\alpha_{\mathrm{D}}$. The $Z \rightarrow h_{\mathrm{D}} A^{\prime}$ branching fraction in Eq. (13) depends linearly on $\alpha_{\mathrm{D}}$ and quadratically on $\varepsilon$, and so the reach in $\varepsilon$ for other values of $\alpha_{\mathrm{D}}$ can be obtained by rescaling our limits on $\varepsilon$ by $1 / \sqrt{\alpha_{\mathrm{D}}}$.

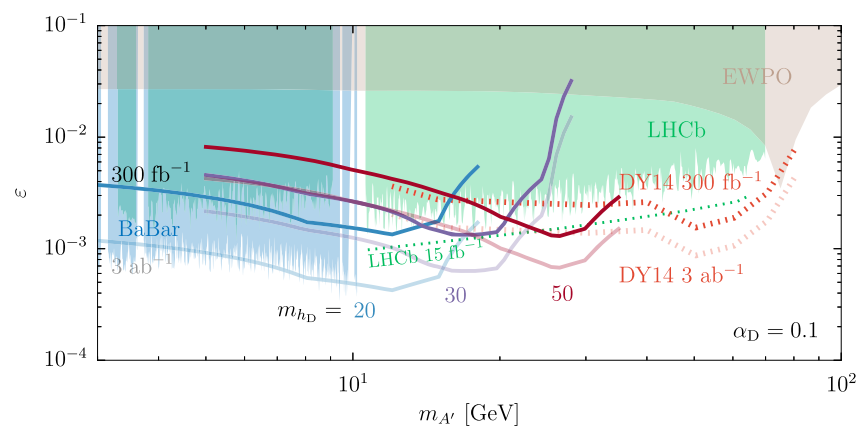

FIG. 4. Sensitivity projections for the $Z \rightarrow h_{\mathrm{D}} A^{\prime} \rightarrow 4 \ell+X$ search for integrated luminosity of $300 \mathrm{fb}^{-1}$ (upper dark lines) and $3000 \mathrm{fb}^{-1}$ (lower faint lines) at $\sqrt{s}=13 \mathrm{TeV}$ and $\alpha_{\mathrm{D}}=0.1$. The dotted green line shows the projected $\mathrm{LHCb}$ sensitivity with $15 \mathrm{fb}^{-1}$ from Ref. [29], while the dashed lines show the projections for the proposed Drell-Yan search from Ref. [32]. Notation and existing bounds are the same as in Fig. 3.

\section{DISPLACED DARK HIGGS DECAYS}

The results of Sec. III focused exclusively on the case where $h_{\mathrm{D}}$ can decay into at least one on-shell $A^{\prime}$. In this section we study the opposite mass hierarchy: $m_{h_{\mathrm{D}}}<m_{A^{\prime}}$. In this regime, the decays $Z \rightarrow A^{\prime} h_{\mathrm{D}}$ are still allowed for $m_{Z}>m_{A^{\prime}}+m_{h_{\mathrm{D}}}$, but the $h_{\mathrm{D}}$ can only decay radiatively or via entirely off-shell $A^{\prime}$. Consequently, the $h_{\mathrm{D}}$ decay is typically displaced from the primary interaction point. These signatures benefit from smaller backgrounds compared to the prompt final states described in Sec. III. We now discuss the salient phenomenological features.

In the parameter space $m_{h_{\mathrm{D}}}<m_{A^{\prime}}$, the $h_{\mathrm{D}}$ can still decay into a two-body final state. For one possibility, $h_{\mathrm{D}}$ decays through a loop of virtual $A^{\prime}$ into SM fermions. This partial width scales approximately as [13]

$$
\begin{aligned}
\Gamma\left(h_{\mathrm{D}} \rightarrow f \bar{f}\right) & \sim \frac{\alpha^{2} Q_{f}^{4} \alpha_{\mathrm{D}} \varepsilon^{4}}{32 \pi^{2}}\left(\frac{m_{f}}{m_{A^{\prime}}}\right)^{2} m_{h_{\mathrm{D}}} \\
& \sim\left(1 \mathrm{~m}^{-1}\right)\left(\frac{\alpha_{\mathrm{D}}}{0.1}\right)\left(\frac{\varepsilon}{10^{-2}}\right)^{4}\left(\frac{15 \mathrm{GeV}}{m_{A^{\prime}}}\right)
\end{aligned}
$$

when summing over SM final-state fermions and taking $m_{A^{\prime}} \sim m_{h_{\mathrm{D}}}$. At this order of $\varepsilon^{4}$, there is also a tree-level fourbody decay of the $h_{\mathrm{D}}$ via two off-shell $A^{\prime}$. However, for the moderate dark Higgs masses that we are interested in, the loop decay typically dominates.

So far, we have assumed that the mixing between the $h_{\mathrm{D}}$ and the SM Higgs is zero, and consequently the only allowed $h_{\mathrm{D}}$ decay modes are via its coupling with $A^{\prime}$. However, a mixing between the $h_{\mathrm{D}}$ and the SM Higgs is induced by a loop of dark vectors. This mixing is given by

$$
V\left(H, H_{\mathrm{D}}\right) \supset \kappa(\mu)|H|^{2}\left|H_{\mathrm{D}}\right|^{2},
$$

where the renormalization-scale-dependent mixing term scales as

$$
\kappa(\mu) \sim \frac{\alpha \alpha_{\mathrm{D}} \varepsilon^{2}}{(4 \pi)^{2}} \log \left(\frac{\mu}{\Lambda}\right)+\kappa(\Lambda)
$$

where $\Lambda$ is an ultraviolet (UV) energy scale. The decay $h_{\mathrm{D}} \rightarrow f \bar{f}$ due to the mixing in Eq. (20) is parametrically similar to the $A^{\prime}$-loop-induced and four-body decay modes. The precise value of this loop-induced mixing depends explicitly on the UV value of $\kappa$. The mixing $\kappa$ can in principle be zero in the infrared, but this represents a tuning of model parameters. Thus, the decay width of $h_{\mathrm{D}}$ for $m_{A^{\prime}}>m_{h_{\mathrm{D}}}$ (and whether it proceeds radiatively or via Higgs mixing) depends sensitively on the value of $\kappa$ in the UV and its renormalization-group evolution.

Because of this model dependence, we take a bottom-up approach and focus on the plausible signatures of $h_{\mathrm{D}}$ decay for $m_{h_{\mathrm{D}}}<m_{A^{\prime}}$, all of which feature decays of $h_{\mathrm{D}}$ at a 
displaced vertex for the parameter space that is accessible to the LHC. The dominant displaced signatures that can arise are as follows:

(i) Displaced decay of $h_{\mathrm{D}}$ into SM fermions according to Eq. (18). The branching fractions into heavyflavor objects $(b, c$, and $\tau$ ) are comparable: the color factors and/or heavier masses of the $b / c$ are compensated by the smaller electric charges. This case will arise when the branching ratio given by Eq. (18) dominates. The $h_{\mathrm{D}}$ will then give (at least) two displaced tracks, which can be leptons or hadrons. The combined final state from the rare $Z$ decay is two prompt leptons in association with the two or more displaced tracks.

(ii) Displaced decay of $h_{\mathrm{D}}$ through Higgs mixing in Eq. (19). This decay occurs predominantly into bottom quarks. We require displaced tracks from the $b$-quark hadronization but apply no $b$-tagging requirements. The displaced vertex comes in association with prompt leptons from the $A^{\prime}$ decay in $Z \rightarrow A^{\prime} h_{\mathrm{D}} \rightarrow \ell^{+} \ell^{-} h_{\mathrm{D}}$.

(iii) The subdominant decay of $h_{\mathrm{D}}$ into four SM fermions can give a rather striking final state; we do not consider it further, although it could give rise to an interesting signature for future study.

We estimate the sensitivity for a prompt, two-lepton final state in association with displaced tracks (leptons or hadrons) originating from a displaced vertex as follows. The signal events are selected using standard dilepton triggers [52]:

(i) two OSSF muons with $p_{\mathrm{T}}>17,8 \mathrm{GeV}$, or

(ii) two OSSF electrons with $p_{\mathrm{T}}>23,12 \mathrm{GeV}$.

We further require the track transverse impact parameters, $\left|d_{0}\right|$, to lie within $1 \mathrm{~mm}<\left|d_{0}\right|<200 \mathrm{~mm}$ (motivated collectively by the ATLAS [60] and CMS [61] $\left|d_{0}\right|$ reconstruction capability). We further require the point of $h_{\mathrm{D}}$ decay to occur within $200 \mathrm{~mm}$ of the primary vertex in both the transverse and the longitudinal directions. Finally, we apply an efficiency for selection of a displaced vertex. This efficiency depends on the details of the experimental search, and in particular the need to reject certain backgrounds. In existing searches, the efficiencies for displaced vertices in the inner detector vary widely from $\sim 10$ to $30 \%$ [60] through to $~ 50 \%$ [61]. The signal also features a resonant $A^{\prime}$ mass that can be reconstructed in the prompt leptons: therefore, backgrounds are lower than for inclusive displaced vertex searches, and data-driven background estimation is more straightforward in the variable $m_{\ell^{+} \ell^{-}}$from the prompt leptons. Therefore, we prioritize signal efficiency and choose to apply a flat $50 \%$ vertex tagging efficiency. The sensitivity of this search is estimated by requiring an observation of 10 signal events, assuming no background.

In Fig. 5 we show the projected sensitivity in two ways. In the top panel we compute the sensitivity to $\varepsilon$ in the
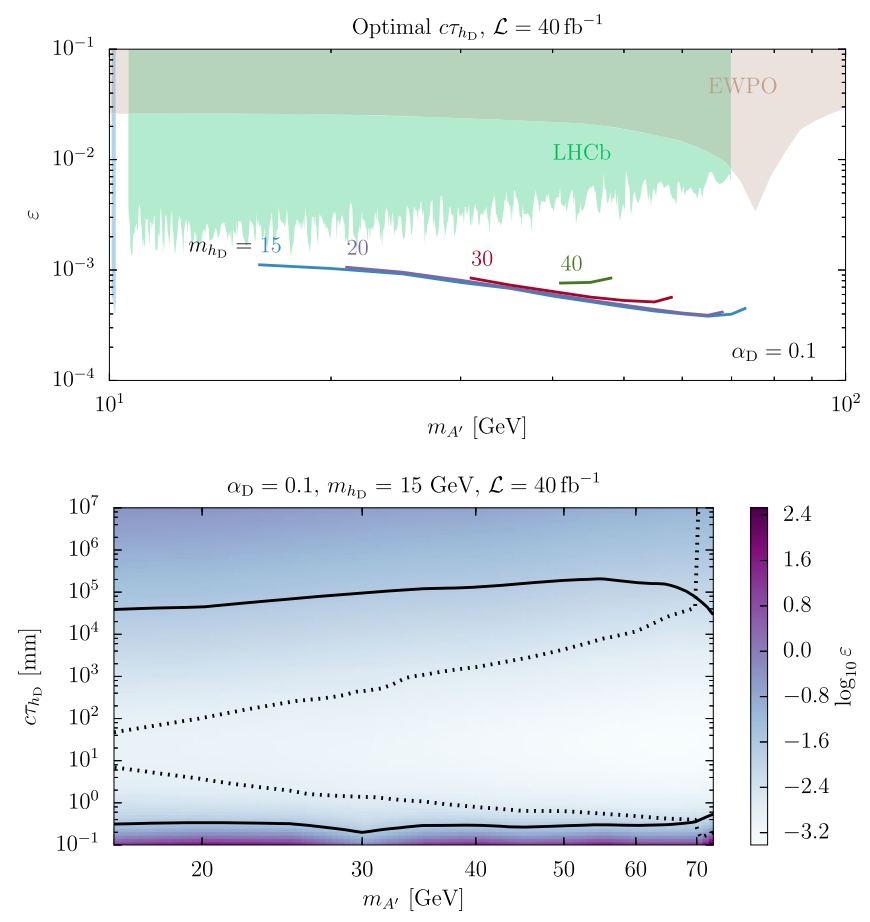

FIG. 5. Projected sensitivity to the scenario where $m_{h_{\mathrm{D}}}<m_{A^{\prime}}$, which gives rise to displaced decays of the dark Higgs. Projections are shown for $\sqrt{s}=13 \mathrm{TeV}$ and $\mathcal{L}=40 \mathrm{fb}^{-1}$. In the top panel, we compute the sensitivity for 10 signal events after cuts to $\varepsilon$ in the $\left(m_{A^{\prime}}, \varepsilon\right)$ plane for different values of $m_{h_{\mathrm{D}}}$ (labeled in $\mathrm{GeV}$ ); for each $m_{A^{\prime}}$ point we have selected the $h_{\mathrm{D}}$ lifetime that gives the optimal reach in $\varepsilon$. In the bottom panel we compute the expected sensitivity in the $\left(m_{A^{\prime}}, c \tau_{h_{\mathrm{D}}}\right)$ plane for $m_{h_{\mathrm{D}}}=15 \mathrm{GeV}$. The projected reach of the displaced search exceeds the existing constraints from electroweak precision observables ( $\mathrm{LHCb}$ prompt search, showing the envelope of the exclusion contour) within the solid (dotted) black contours.

$\left(m_{A^{\prime}}, \varepsilon\right)$ plane, where for each choice of masses we have selected the $h_{\mathrm{D}}$ lifetime that gives the optimal reach in $\varepsilon$. In the bottom panel we select a specific value of $m_{h_{\mathrm{D}}}$ and show the expected sensitivity in the $\left(m_{A^{\prime}}, c \tau_{h_{\mathrm{D}}}\right)$ plane. The projected sensitivity of this search exceeds the current limits from EWPO (LHCb) inside the solid black (dotted) contours. With current levels of data, one can already probe $\varepsilon$ at or better than the level of $10^{-3}$.

\section{DISCUSSION AND CONCLUSIONS}

In this article, we have shown that rare decays of the Standard Model $Z$ boson are powerful probes of hidden sectors. Using a dark Abelian Higgs model as a benchmark, we have demonstrated how studying rare $Z$ decays into hidden sector particles can allow for the discovery of exotic particles through the same interactions responsible for generating hidden-sector particle masses.

$Z$ decays into hidden-sector particles typically give rise to large multiplicities of soft particles. When the 
hidden-sector particles can decay leptonically, such as in the dark Abelian Higgs scenario, this results in striking events with up to six soft leptons. We have demonstrated a range of prompt and displaced signatures that can occur in this model, providing a path for experimentally discovering or constraining these new particles.

Crucial to the success of the strategies we outline in this article is the fact that trigger and reconstruction thresholds for leptons must be kept low. Because the $Z$ boson mass energy is distributed among six or more particles, a moderate increase in trigger thresholds would greatly curtail the sensitivity of ATLAS or CMS to high-multiplicity hidden-sector signatures. While the high-luminosity running of the LHC will introduce new challenges such as increased pileup, we urge the experimental collaborations to consider maintaining low-threshold, high-multiplicity triggers where possible (including potential triggers requiring four or more leptons) in order to retain sensitivity to well-motivated hidden-sector models. Furthermore, our study has focused on only one particular high-multiplicity hidden-sector signature: models giving rise to even higher multiplicities are possible, motivating keeping high-multiplicity trigger thresholds as low as possible.

The searches we propose are complementary to existing proposals for observation of the direct production of the dark photon, $A^{\prime}$. A discovery in both channels could allow for the determination of the $A^{\prime}$ mixing with the SM, the hidden-sector gauge coupling, and the masses of the dark photon and dark Higgs. A comprehensive characterization of the properties of the hidden sector is therefore possible at the LHC.

Finally, we have focused on the specific case where the $Z$ boson can decay to a dark gauge boson and a dark scalar where both are on shell. There exist spectra where this is not the case, such as when $m_{Z}<m_{A^{\prime}}+m_{h_{\mathrm{D}}}$, and there may yet be other interesting signatures of dark Higgs-strahlung originating from off-shell $Z$ production. Indeed, the signatures we discuss in this article should be applicable to more general mass hierarchies, and we encourage ATLAS, $\mathrm{CMS}$, and $\mathrm{LHCb}$ to avoid overly optimizing their search strategies to the particular examples studied here where possible.

\section{ACKNOWLEDGMENTS}

We would like to thank Justin Chiu, Michel Lefebvre, Michael Peskin, and Maxim Pospelov for helpful conversations. E. I. is supported by the United States Department of Energy under Grant Contract No. desc0012704. N. B. is supported by the United States Department of Energy under Contract No. DE-AC02-76SF00515. We express our gratitude to the Aspen Center for Physics, where part of this work was completed and which is supported by National Science Foundation Grant No. PHY-1607761.
[1] C. H. Chen, M. Drees, and J. F. Gunion, Phys. Rev. Lett. 76, 2002 (1996).

[2] S. D. Thomas and J. D. Wells, Phys. Rev. Lett. 81, 34 (1998).

[3] C. Han, A. Kobakhidze, N. Liu, A. Saavedra, L. Wu, and J. M. Yang, J. High Energy Phys. 02 (2014) 049.

[4] M. Low and L.-T. Wang, J. High Energy Phys. 08 (2014) 161.

[5] J. Alwall, M.-P. Le, M. Lisanti, and J. G. Wacker, Phys. Rev. D 79, 015005 (2009).

[6] J. Fan, M. Reece, and J. T. Ruderman, J. High Energy Phys. 11 (2011) 012.

[7] J. A. Evans and Y. Kats, J. High Energy Phys. 04 (2013) 028.

[8] C. Boehm and P. Fayet, Nucl. Phys. B683, 219 (2004).

[9] M. Pospelov, Phys. Rev. D 80, 095002 (2009).

[10] M. J. Strassler and K. M. Zurek, Phys. Lett. B 651, 374 (2007).

[11] M. J. Strassler and K. M. Zurek, Phys. Lett. B 661, 263 (2008).

[12] T. Han, Z. Si, K. M. Zurek, and M. J. Strassler, J. High Energy Phys. 07 (2008) 008.

[13] B. Batell, M. Pospelov, and A. Ritz, Phys. Rev. D 79, 115008 (2009).
[14] B. Batell, M. Pospelov, and A. Ritz, Phys. Rev. D 83, 054005 (2011).

[15] N. Arkani-Hamed and N. Weiner, J. High Energy Phys. 12 (2008) 104.

[16] C. Cheung, J. T. Ruderman, L.-T. Wang, and I. Yavin, J. High Energy Phys. 04 (2010) 116.

[17] M. Baumgart, C. Cheung, J. T. Ruderman, L.-T. Wang, and I. Yavin, J. High Energy Phys. 04 (2009) 014.

[18] D. Curtin et al., Phys. Rev. D 90, 075004 (2014).

[19] G. Aad et al. (ATLAS Collaboration), J. High Energy Phys. 11 (2014) 088.

[20] G. Aad et al. (ATLAS Collaboration), J. High Energy Phys. 02 (2016) 062.

[21] V. Khachatryan et al. (CMS Collaboration), Phys. Lett. B 752, 146 (2016).

[22] T. A. collaboration (ATLAS Collaboration), CERN, Technical Report No. ATLAS-CONF-2017-060, 2017.

[23] CERN, Technical Report No. CMS-PAS-SUS-16-052, 2017.

[24] G. Aad et al. (ATLAS Collaboration), Phys. Lett. B 743, 15 (2015).

[25] R. Aaij et al. (LHCb Collaboration), Eur. Phys. J. C 77, 224 (2017).

[26] B. Holdom, Phys. Lett. 166B, 196 (1986). 
[27] P. Galison and A. Manohar, Phys. Lett. 136B, 279 (1984).

[28] I. Hoenig, G. Samach, and D. Tucker-Smith, Phys. Rev. D 90, 075016 (2014).

[29] P. Ilten, Y. Soreq, J. Thaler, M. Williams, and W. Xue, Phys. Rev. Lett. 116, 251803 (2016).

[30] F. Elahi and A. Martin, Phys. Rev. D 93, 015022 (2016).

[31] H. Davoudiasl, H.-S. Lee, I. Lewis, and W. J. Marciano, Phys. Rev. D 88, 015022 (2013).

[32] D. Curtin, R. Essig, S. Gori, and J. Shelton, J. High Energy Phys. 02 (2015) 157.

[33] J. P. Chou, D. Curtin, and H. J. Lubatti, Phys. Lett. B 767, 29 (2017).

[34] J. Liu, X.-P. Wang, and F. Yu, J. High Energy Phys. 06 (2017) 077.

[35] B. W. Lee and S. Weinberg, Phys. Rev. Lett. 39, 165 (1977).

[36] M. Pospelov, A. Ritz, and M. B. Voloshin, Phys. Lett. B 662, 53 (2008).

[37] M. Cvetic and P. Langacker, Mod. Phys. Lett. A 11, 1247 (1996).

[38] S. A. Abel, M. D. Goodsell, J. Jaeckel, V. V. Khoze, and A. Ringwald, J. High Energy Phys. 07 (2008) 124.

[39] M. Goodsell, J. Jaeckel, J. Redondo, and A. Ringwald, J. High Energy Phys. 11 (2009) 027.

[40] J. P. Lees et al. (BABAR Collaboration), Phys. Rev. Lett. 113, 201801 (2014).

[41] J. P. Lees et al. (BABAR Collaboration), Phys. Rev. Lett. 108, 211801 (2012).

[42] I. Jaegle (Belle Collaboration), Phys. Rev. Lett. 114, 211801 (2015).

[43] A. Hook, E. Izaguirre, and J. G. Wacker, Adv. High Energy Phys. 2011, 859762 (2011).

[44] M. Awramik, M. Czakon, A. Freitas, and G. Weiglein, Phys. Rev. D 69, 053006 (2004).
[45] S. Chatrchyan et al. (CMS Collaboration), J. High Energy Phys. 12 (2013) 030.

[46] J. Jaeckel, M. Jankowiak, and M. Spannowsky, Phys. Dark Universe 2, 111 (2013).

[47] R. Aaij et al. (LHCb Collaboration), arXiv:1710.02867.

[48] G. Aad et al. (ATLAS Collaboration), Phys. Rev. D 92, 092001 (2015).

[49] CERN, Technical Report No. ATL-DAQ-PUB-2017-001, 2017.

[50] V. Khachatryan et al. (CMS Collaboration), Phys. Lett. B 740, 250 (2015); 757, 569(E) (2016).

[51] V. Khachatryan et al. (CMS Collaboration), Eur. Phys. J. C 74, 3036 (2014).

[52] A. M. Sirunyan et al. (CMS Collaboration), arXiv: 1709.05406.

[53] J. Alwall, R. Frederix, S. Frixione, V. Hirschi, F. Maltoni, O. Mattelaer, H. S. Shao, T. Stelzer, P. Torrielli, and M. Zaro, J. High Energy Phys. 07 (2014) 079.

[54] J. Alwall, S. de Visscher, and F. Maltoni, J. High Energy Phys. 02 (2009) 017.

[55] T. Sjostrand, S. Mrenna, and P. Z. Skands, J. High Energy Phys. 05 (2006) 026.

[56] T. Sjostrand, S. Mrenna, and P. Z. Skands, Comput. Phys. Commun. 178, 852 (2008).

[57] T. Sjostrand, S. Ask, J. R. Christiansen, R. Corke, N. Desai, P. Ilten, S. Mrenna, S. Prestel, C. O. Rasmussen, and P. Z. Skands, Comput. Phys. Commun. 191, 159 (2015).

[58] A. L. Read, J. Phys. G 28, 2693 (2002).

[59] S. Chatrchyan et al. (CMS Collaboration), Phys. Lett. B 726, 564 (2013).

[60] G. Aad et al. (ATLAS Collaboration), Phys. Rev. D 92, 072004 (2015).

[61] V. Khachatryan et al. (CMS Collaboration), Phys. Rev. D 91, 052012 (2015). 